\title{
Evaluation of endoscopic cartilage myringoplasty in management of dry central perforation
}

\author{
Original Abdellatif Ibrahim El-Rashidy ${ }^{l}$,Essam Abdelwanees Behiry ${ }^{l}$, Ashraf Ali El-Demerdash ${ }^{1}$, \\ Article Wael Fahmy Elkholy ${ }^{2}$ \\ Department of Otorhinolaryngology, ${ }^{1}$ Faculty of Medicine, Menoufia University, ${ }^{2}$ Shebin \\ El-Kom Teaching Hospital, Menoufia, Egypt
}

\begin{abstract}
Background: Tubotympanic type of chronic suppurative otitis media is one of the most frequent diseases seen in ENT clinics. On examination, central perforation in the tympanic membrane can be seen. Different approaches were used for closure of tympanic membrane perforations mainly the postauricular, the end aural and trans canal.

Objective: To compare between endoscopic and microscopic trans canal myringoplasty using tragal cartilage graft regarding success rate and postoperative hearing improvement.

Patients and Methods: This prospective study was carried out on 24 patients from December 2017 till August 2018. All patients were presented with small or medium sized central perforation which was dry for at least 3 months, and then subjected to preoperative clinical, endoscopic, audiological assessment. Patients were divided equally and randomly into two groups, group A for endoscopic myringoplasty and group B for microscopic myringoplasty, composite cartilageperichondrium graft was used for both groups and all of them were arranged to postoperative re-evaluation after $1,2,4$ and 12 weeks.

Results: The mean age group of our studied patients was $31.5 \pm 7$ years. (45.8 \%) of patients were male, while (54.2\%) of them were female. Graft uptake in endoscopic myringoplasty was $100 \%$ while in microscopic was $83 \%$ of cases. Postoperative air bone gap was less than $10 \mathrm{~dB}$ in $100 \%$ of the patients in endoscopic group and $66.67 \%$ in microscopic group.

Conclusion: Although these results were statistically insignificant, advantages of endoscopic myringoplasty overcomes most of the disadvantage of microscope.
\end{abstract}

Key Words: Cartilage graft, central perforation, endoscopic myringoplasty, tympanic membrane

Received: 05 January 2019, Accepted: 10 May 2019.

Corresponding Author: Wael Fahmy Elkholy, Department of Otorhinolaryngology, Shebin El-Kom Teaching Hospital, Menoufia Governorate, Egypt, Tel.:+201014898986, E-mail: waelfahmy22588@gmail.com

ISSN: 2090-0740, November 2019 Vol.20, No.3

\section{INTRODUCTION}

The introduction of tympanoplasty by Zöllner and Wullstein, was in 1952 and numerous graft materials and methods of placement have been described to close the perforations of the tympanic membrane ${ }^{[1]}$. Different approaches were used for closure of tympanic membrane perforations mainly the postauricular, the end aural and trans canal with placement of the harvested graft. The most commonly used graft materials are the temporalis fascia, perichondrium, cartilage and fat plugs specially for small perforations ${ }^{[2]}$ The role of endoscopes in otology as a diagnostic, surgical and teaching tool is increasingly recognized because of their superior optical properties and their capacity to visualize hidden areas with minimal invasion of tissues as compared to a microscope. Their use either as an exclusive equipment or as an adjunct to microscope in otology practice is growing very slowly but appears promising ${ }^{[3]}$.
Increase bilirubin level, one of the most common risk factor associated with hearing loss. In utero infections are another common risk factor associated with hearing loss according to JCIH, 2007 $7^{[2]}$. What about in Egypt specifically Upper Egypt.

This is a comparative observational (cohort) prospective study. After getting a written consent from all enrolled patients, this study was carried on 24 patients (24 ears) diagnosed to have uncomplicated tubotympanic type of chronic suppurative otitis media who underwent myringoplasty. Their age was ranging from 16 to 56 years old. Patients were selected from out patients' clinic of Otolaryngology Department in Menoufia University Hospital from December 2017 to August 2018. Patients of this study had complaining inactive mucosal unilateral CSOM of small or medium sized central perforation, we considered perforations occupying one quadrant or $\leq 25 \%$ of the TM surface area to be small 
sized perforation and that occupying two quadrants or $\leq 50 \%$ of the TM surface area to be medium sized perforation. no middle ear pathology, no mucosal changes, dry for at least 3 months without any episode of otorrhea and a pure conductive hearing loss of air bone gap $\leq 35 \mathrm{dbs}$. Patients with circumferentially narrow canal, marginal or attic perforations, retraction pocket and actively discharging ear, Sensorineural hearing loss (SNHL) and AB gap more than $35 \mathrm{~dB}$, Revision cases or patients suffering from chronic rhinosinusitis, adenoids, untreated medical conditions such as otomycosis, uncontrolled diabetes mellitus, and upper respiratory tract infection were excluded from this study. All patients were subjected to preoperative full history taking, full ENT clinical examination, rigid otooendoscopic assessment, Basic audiological assessment. The patients of this study then were divided equally and randomly into two groups, group A for endoscopic myringoplasty and group B for microscopic myringoplasty, and all of them were arranged to postoperative re-evaluation clinically, otoendoscopically and by evaluation tests after 1, 2, 4, 12 weeks.

\section{Operative technique}

General anesthesia was used for all cases (24 patients). Hypotensive anesthesia was done in which the patient blood pressure was near $90 / 60 \mathrm{mmHg}$ and the pulse about $60 / \mathrm{min}$. Zero-degree shenda endoscope $(2.7 \mathrm{~mm}$ in diameter and $11 \mathrm{~cm}$ in length) was used and all surgeries were done by visualization using the monitor. All endoscopic myringoplasties were done through the Trans canal approach without the use of microscope.

During the use of the Endoscope, the monitor is placed facing the surgeon according to the operated ear. The surgeon holds the camera together with the endoscope with his non-dominant hand (usually the left hand). The patient lies flat on his back with his head turned 45 degree opposite the operated ear. The view is controlled by the direction of the endoscope by the non-dominant hand. The surgical steps were done using the dominant hand (usually the right hand). The instruments precede the tip of the endoscope during surgery by about $2-4 \mathrm{~mm}$. Endoscopic view of tympanic membrane perforation. Local injection of xylocaine and 1/200,000 adrenaline at cartilaginous bony junction of ear canal at 3, 6, 9, 12 o'clock, and the tragus. Graft harvesting by a small incision is made in the posterior aspect of the tragus and the cartilage with the perichondrium is exposed. Piece of cartilage is excised with perichondrium on both two faces. The graft is then treated; the layer of perichondrium that covered the cartilage on the convex side is gently removed, stripping the cartilage from this side. The perichondrium of the concave surface of the cartilage is retained. The cartilage is thinned using a sharp blade. The graft is left to dry and stitches are used to close skin of tragus. Refreshment of the margins of the perforation using straight sharp needle. Incision in the posterior canal wall starting from 6 to 12 o'clock at about $1 \mathrm{~cm}$ from annulus. Then we elevate the tympanomeatal flap (TMF) and reflect it anteriorly. Then graft is placed using underlay technique with perichondrium facing laterally. The graft is supported in position by a disc of gel foam over the promontory. The tympanomeatal flap is repositioned and good apposition of the graft with the tympanic membrane is checked through the endoscope. Then gelfoam is placed in external auditory canal to stabilize the graft. In microscopic group, surgeries were done under general anesthesia Classical myringoplasty using microscope was done through per meatal approach utilizing similar incision and same steps as previously described.

\section{statistical analysis}

Statistical calculations and data analysis of this study was done using Statistical Package for the Social Sciences (SPSS V 17), IBM Corp, Armonk, NY, USA. Mean value and Standard Deviation (SD) were used for quantitative data, and Frequency and percentage for qualitative data. Paired Student T-test was used to compare between related samples, while Unpaired Student T-test was used to compare between two groups in quantitative data. Chi square test was used to compare between two independent qualitative variables

\section{RESULTS}

A total of 24 patients included in this study were between 16-56 years old, the mean age group of the patients was $(31.5 \pm 7)$. In this study, there were 11 males and 13 females. 9 patients had perforation in the right ear, while 15 patients had perforation in the left ear.

According to the size of perforation, there were 14 $(58.2 \%)$ patients with medium sized perforation, while 10 $(41.8 \%)$ patients with small sized perforation. According to the site of perforation, the 14 cases with medium sized perforation were divided as following: 6 patients with anterior perforation, 4 patents with posterior perforation, while 4 patients with inferior perforation. $(P$-value $=0.717)$ which is statistically insignificant. The 10 cases with small sized perforation were divided as following: 1 patient with Anterosuperior perforation, 4 patients withAnteroinferior perforation, 2 patients with posterosuperior perforation, while 3 patients with posteroinferior perforation. $(P$-value $=0.721)$ which is statistically insignificant (Table 1) 
Table 1: Size and Site of perforation in cases

\begin{tabular}{|c|c|c|c|c|c|c|c|}
\hline \multirow{3}{*}{ Size of perforation } & \multirow{3}{*}{ Site of perforation } & \multicolumn{4}{|c|}{$\begin{array}{l}\text { Patients with dry central } \\
\text { perforation }=24\end{array}$} & \multirow{3}{*}{$\chi^{2}$ test } & \multirow{3}{*}{$P$-value } \\
\hline & & \multicolumn{2}{|c|}{ Group A } & \multicolumn{2}{|c|}{ Group B } & & \\
\hline & & No. & $\%$ & No. & $\%$ & & \\
\hline \multirow{3}{*}{$\begin{array}{l}\text { Medium sized } \\
\text { (14 ears, } 58.2 \% \text { ) }\end{array}$} & Anterior & 4 & $16.6 \%$ & 2 & $8.3 \%$ & \multirow{3}{*}{2.105} & \multirow{3}{*}{0.717} \\
\hline & Posterior & 1 & $4.2 \%$ & 3 & $12.5 \%$ & & \\
\hline & Inferior & 2 & $8.3 \%$ & 2 & $8.3 \%$ & & \\
\hline \multirow{5}{*}{$\begin{array}{l}\text { Small sized } \\
\text { (10 ears, } 41.8 \%)\end{array}$} & Anterosuperior & 0 & 0 & 1 & $4.2 \%$ & \multirow{5}{*}{2.079} & \multirow{5}{*}{0.721} \\
\hline & Anteroinferior & 3 & $12.5 \%$ & 1 & $4.2 \%$ & & \\
\hline & & & & & & & \\
\hline & Posterosuperior & 1 & $4.2 \%$ & 1 & $4.2 \%$ & & \\
\hline & Posteroinferior & 1 & $4.2 \%$ & 2 & $8.3 \%$ & & \\
\hline
\end{tabular}

$\chi 2=$ chi-square test, $P$ value: $\mathrm{NS}=$ Non-significant $(P$-value $>0.05), \mathrm{S}=\operatorname{significant}(P$-value $\leq 0.05 \mathrm{HS}=$ highly significant $(P$-value $\leq 0.001)$.

According to success rate, in group A 12 cases (100\%) the graft was taken and the tympanic membrane defect was completely closed, while in group B 10 cases $(83.3 \%)$ and there were 2 failed cases. $(P$-value $=0.140)$ which is not statistically significant (Table 2 ).

Table 2: Success rate results

\begin{tabular}{ccccccc}
\hline \multirow{2}{*}{$\begin{array}{c}\text { Graft } \\
\text { uptake }\end{array}$} & \multicolumn{2}{c}{ Group A } & \multicolumn{2}{c}{ Group B } & $\begin{array}{c}\chi^{2} \\
\text { test }\end{array}$ & $P$-value \\
\cline { 2 - 6 } & No. & $\%$ & No. & $\%$ & & \\
\hline Taken & 12 & $100 \%$ & 10 & $83.3 \%$ & & \\
Failed & 0 & 0 & 2 & $16.7 \%$ & 6.930 & 0.140
\end{tabular}

$\chi^{2}=$ chi-square test, $P$ value: $\mathrm{NS}=$ Non-significant $(P$-value $>0.05), \mathrm{S}=$ significant $(P$-value $\leq 0.05 \mathrm{HS}=$ highly significant $(P$-value $\leq 0.001)$.

According to preoperative air bone gap, in group $\mathrm{A}$ the $\mathrm{ABG}$ was between $10-20 \mathrm{~dB}$ in 2 cases, between 20-30 dB in 9 cases, and between 30-35 dB in 1 case. The mean $\mathrm{ABG}$ in group $\mathrm{A}$ was $24.1(\mathrm{SD} \pm 4.8)$. in group $\mathrm{B}$ the $\mathrm{ABG}$ was between $10-20 \mathrm{~dB}$ in 2 cases, between 20-30 dB in 7 cases, and between 30-35 dB in 3 case. The mean $A B G$ in group A was 24.7 ( $\mathrm{SD} \pm 4.6$ ). The $P$-value is 0.764 which is statistically insignificant (Table 3).
Table 3: Preoperative Air-Bone Gap (ABG)

\begin{tabular}{|c|c|c|c|c|}
\hline \multirow[b]{2}{*}{ Group Range } & \multicolumn{2}{|c|}{$\begin{array}{l}\text { Patients with dry central } \\
\text { perforation }=24\end{array}$} & \multirow{2}{*}{ t-test } & \multirow{2}{*}{$P$-value } \\
\hline & Group A & Group B & & \\
\hline $10-20 \mathrm{~dB}$ & 2 & 2 & \multirow{4}{*}{0.305} & \multirow{4}{*}{0.764} \\
\hline $20-30 \mathrm{~dB}$ & 9 & 7 & & \\
\hline $30-35 \mathrm{~dB}$ & 1 & 3 & & \\
\hline Mean \pm SD & $24.1 \pm 4.8$ & $24.7 \pm 4.6$ & & \\
\hline
\end{tabular}

$P$ value: $\mathrm{NS}=$ Non-significant $(P$-value $>0.05), \mathrm{S}=$ significant $(P$-value $\leq 0.05 \mathrm{HS}=$ highly significant $(P$-value $\leq 0.001)$.

According to postoperative air bone gap, in group A the ABG was between $0-10 \mathrm{~dB}$ in all 12 cases. The mean $\mathrm{ABG}$ in group $\mathrm{A}$ was $4.8(\mathrm{SD} \pm 2.4)$. In group $B$ the $A B G$ was between $0-10 \mathrm{~dB}$ in 8 cases, and between $10-20 \mathrm{~dB}$ in 4 cases. The mean $\mathrm{ABG}$ in group $\mathrm{A}$ was $8.5(\mathrm{SD} \pm 5.7)$. $(P$-value $=0.048)$ which is statistically significant (Table 4). 
Table 4: Postoperative (3monthes) Air-Bone Gap (ABG)

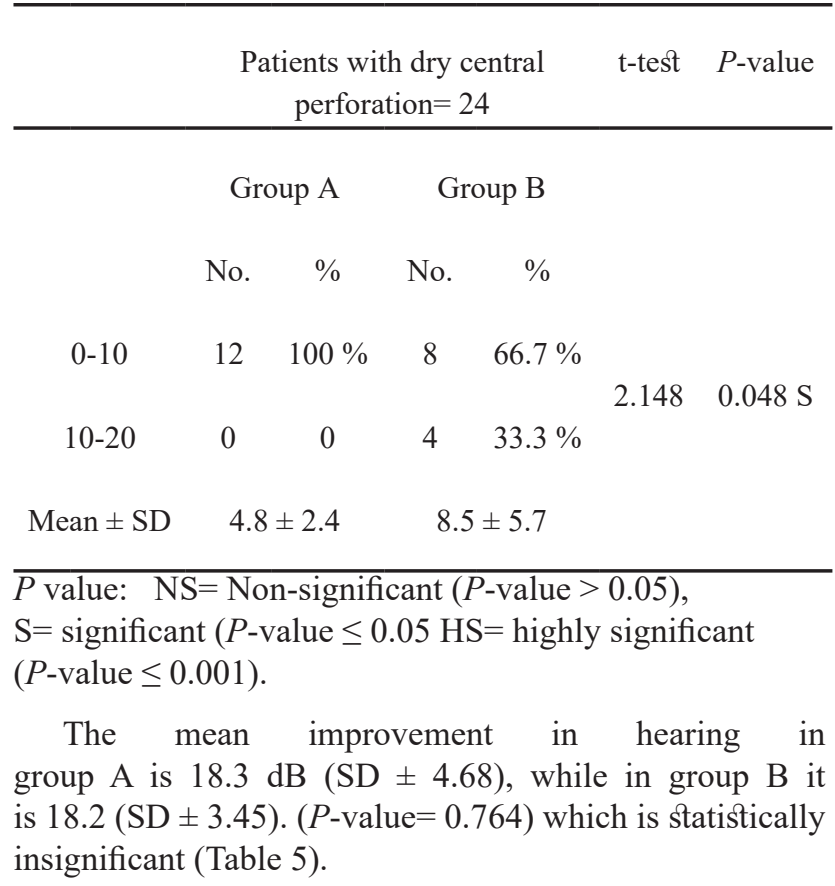

Table 5: The mean improvement of hearing after 3 months

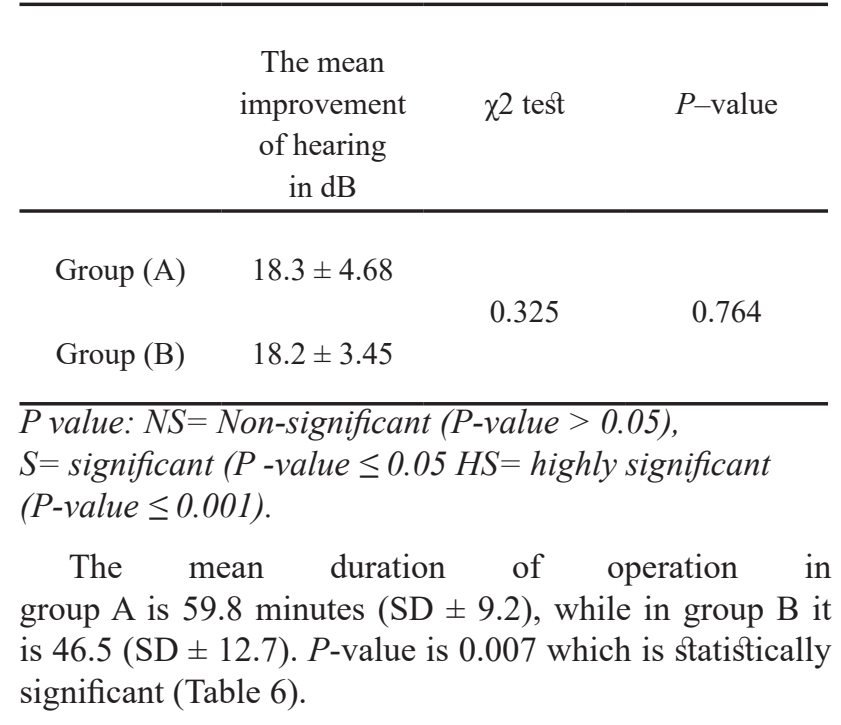

Table 6: Duration of the operation

Duration of the operation in minutes $( \pm \mathrm{SD}) \quad$ t-test $\quad P$-value

\begin{tabular}{llll}
\hline Group (A) & $59.8 \pm 9.2$ & 3.099 & $0.007 \mathrm{~S}$ \\
Group (B) & $46.5 \pm 12.7$ & & \\
\hline
\end{tabular}

$\chi^{2}=$ chi-square test $P$ value: $N S=$ Non-significant (P-value $>0.05), S=$ significant $(P$-value $\leq 0.05 \mathrm{HS}=$ highly significant $(P$-value $\leq 0.001)$.

\section{DISCUSSION}

Many ENT surgeons perform myringoplasty under an operating microscope. However, despite providing direct exposure, microscopy may be insufficient in viewing certain areas during surgery. Although there are no exposure problems in the posterior and inferior areas, there may be exposure problems and hidden areas that cannot be seen under a microscope especially most anterior perforations and can be better observed via thin and rigid endoscopes with different angles. In the endoscopic myringoplasty procedure, a thin, rigid endoscope allows for functional reconstruction during surgery and the performance of minimally invasive procedures and conservative surgeries with protection of the anatomy ${ }^{[4]}$.

In sharp contrast, the endoscope brings the surgeon's eye to the tip of the scope. Hence the view through the endoscope will not be restricted by the narrowest segment of the external auditory canal. The wide angle 0 -degree scope visualizes the entire tympanic membrane in one frame. There is no need to frequently adjust the patient's head or do canaloplasty thereby saving operative time. Also, on the economic view, the endoscope is easily transportable and hence is ideal for use in ear surgery camps and cheaper than the microscope that will be difficult in transporting ${ }^{[5]}$.

This study was done on 24 patients had dry central perforation underwent myringoplasty. Age was between 16 to 56 years old. Sex distribution was 11 males and 13 females. Out of 24 cases, 10 cases (41.8\%) were having small T.M. perforation, 14 cases $(58.3 \%)$ were having medium sized T.M. perforation.

In the current study, success rate in endoscopic myringoplasty was $100 \%$ while in microscopic was $83 \%$ of cases. There was no statistically significant difference between results of graft uptake among studied groups.

Yadav SPS. et $a l^{[6]}$ in his study of endoscopic myringoplasty 40 out of the 50 patients had an intact tympanic membrane in the 8 th postoperative week, accounting for an $80 \%$ success rate.

Lade H. et al..$^{[7]}$ in his study to ascertain the feasibility of transcanal endoscopic underlay myringo $\neg$ plasty using cartilage and compare the results with microscopic myringoplasty. A graft uptake rate of $83.3 \%$ was observed in both groups postoperatively after 24 weeks.

El-Guindy ${ }^{[8]}$ has evaluated the role of the rigid endoscope in the management of 36 cases of dry central perforation of the tympanic membrane. The graft uptake rate was $91.7 \%$.

Harugop et al..$^{[5]}$ the graft take rate in endoscopic was $82 \%$, while in microscopic was $86 \%$. While 
Raj et al. ${ }^{[9]}$ the graft take rate in endoscopic was $90 \%$, while in microscopic was $85 \%$.

Patel et $a .^{[10]}$ the graft take rate in endoscopic was $68.18 \%$, while in microscopic was $68.18 \%$. Lakpathi et al. ${ }^{[11]}$ the graft take rate in endoscopic was $88 \%$, while in microscopic was $90 \%$.

Graft up take was dependent on size and site of the perforation; the 2 failed cases were medium sized perforations which are located anteroinferior and performed with microscope.

Although most authors prefer to do postoperative pure tone audiometry after 6 months, many studies showed that there is no significant difference in the results before and after 6 months. 14 In our study, patients undergone postoperative pure tone audiometry after 3 months ${ }^{[10]}$.

Postoperative air bone gap was less than $10 \mathrm{~dB}$ in $100 \%$ of the patients in endoscopic group and $66.67 \%$ in microscopic group. The mean hearing gain was $18.3 \mathrm{~dB}$ $(\mathrm{SD} \pm 4.68)$ in endoscopic group and $18.2 \mathrm{~dB}(\mathrm{SD} \pm 3.45)$ in microscopic group. The above results are consistent with the earlier studies.

Mohindra ${ }^{[3]}$ did 49 cases of myringoplasty and through the trans canal route using rigid endoscopes. The success rate regarding perforation closure was $91.5 \%$ and average air bone gap improvement was $22.24 \mathrm{~dB}$ in the myringoplasty groups.

EL-Guindy ${ }^{[8]}$ has evaluated the role of the rigid endoscope in the management of 36 cases of dry central perforation of the tympanic membrane. The graft uptake rate was $91.7 \%$ and air bone gap was closed to less than $10 \mathrm{~dB}$ in $83.3 \%$. He used endoscope along with the manometry to evaluate the tubal function before ear surgery.

The time of surgery was comparable between the 2 groups, although time in group A was insignificantly longer 59 minutes $(\mathrm{SD} \pm 9.2)$ than that of group $\mathrm{B}$ was 46.5 minutes $(\mathrm{SD} \pm 12.7)$ due to the repeated cleaning and defogging of the tip of the endoscope.

The duration of the operation is an important parameter in terms of the duration of anesthesia, the surgeon's concentration and the increased risk of iatrogenic complications. In a study by Ghaffar et $a l,{ }^{[12]}$ the mean operation duration was 62.85 minutes among 34 patients who underwent microscopic tympanoplasty. Another study reported the average time taken for surgery was less in the endoscopic group $(1 \mathrm{~h})$ as compared to the microscopic group $(2 \mathrm{~h})$. The reason for these differences may be related to the fact that neither suturing nor extra time to view hidden areas is needed during endoscopic procedures ${ }^{[13]}$.
Patel et al. ${ }^{[10]}$ reported that average time taken for endoscopic tympanoplasty was around 75 minutes, while the same time taken for microscopic tympanoplasty was around 90 minutes.

In our study, endoscopic myringoplasty took more time. this is because blood usually soils the tip of endoscope which obscures the surgical field. This problem can be solved by developing a stand for endoscope, which can fix the scope in desired position so both hands are free to operate. This difficulty may also be overcomed by experience and more practice of endoscopic technique. Savlon is used as a defogging agent for endoscopes. Studies on effect of savlon on middle ear mucosa and inner ear are not sufficient, thus safety of savlon is yet to be established ${ }^{[10]}$.

\section{CONCLUSION}

Success rate in endoscopic myringoplasty was $100 \%$ while in microscopic was $83 \%$ of cases. There was no statistically significant difference between results of graft uptake among studied groups.

Graft up take was dependent on size and site of the perforation; the 2 failed cases were medium sized perforations which are located anteroinferior and performed with microscope.

The mean ABG in endoscopic group was $24.1 \mathrm{~dB} \quad(\mathrm{SD} \pm 4.8)$ pre-operatively and $4.8 \mathrm{~dB}(\mathrm{SD} \pm 2.4)$ post-operatively. While the mean $\mathrm{ABG}$ in microscopic group was $24.7 \mathrm{~dB}(\mathrm{SD} \pm 4.6)$ preoperatively and $8.5 \mathrm{~dB}(\mathrm{SD} \pm 5.7)$ post-operatively. The mean hearing gain was $18.3 \mathrm{~dB}(\mathrm{SD}=4.68)$ in endoscopic group and $18.2 \mathrm{~dB}(\mathrm{SD}=3.45)$ in microscopic group.

Although these results were statistically insignificant, panoramic, wide angle, and magnified view provided by endoscope as well as ability to avoid canaloplasty and uninterrupted picture overcomes most of the disadvantage of microscope

The duration of the operation was more in endoscopic than microscopic. This was statistically significant. This happened due to soiling of the tip of endoscope with blood which obscures the surgical field.

Loss of depth perception and one-handed technique are some of the disadvantage of endoscope that can be overcome with practice and use of endo-holder.

Ths, endoscopic myringoplasty can be a good alternative of microscopic myringoplasty. endoscope holds the greatest promise in ear surgery in coming days. 


\section{CONFLICT OF INTEREST}

There are no confilict of interest.

\section{REFERENCES}

1. Cavaliere M, Mottola G, Rondinellini M, Iemma M. (2009) Tragal cartilage in tympanoplasty: anatomic and functional results in 306 cases. Acta Otorhinolaryngol Ital 2009:29:27-32.

2. Rizer FM. (1997). Overlay Versus Underlay Tympanoplasty Part I: Historical Review of the Literature. Laryngoscope; 107:1-25.

3. Mohindra M., Panda N. (2010). Ear surgery without microscope; is it possible? Indian J. Otolaryngology Head and Neck Surgery 62,(2):138-141.

4. Dundar R, Kulduk E, Soy FK, Aslan M, Hanci D, Muluk NB, Cingi C. (2014). Endoscopic Versus Microscopic Approach to Type 1 Tympanoplasty in Children. Int $\mathbf{J}$ Pedia Otorhinolaryngol.; 78:1084-1089. Chaiyasate S, Baron I, Clement P. Analysis of paranasal sinus developmentandanatomical variations: a CT genetic study in twins. Clinical Otolaryngology. 2007; (32):93-7.

5. Harugop A.S, Mudhol R. S., Godhi R. A. (2008). A comparative study of endoscope assisted myringoplasty and microscope assisted myringoplasty. Indian $\mathrm{J}$. Otolaryngology head \& Neck Surgery, 60:29-302.

6. Yadav SPS, Aggarwal N, Julaha M, Goel A. (2009). Endoscope assisted myringoplasty. Singapore Med J;50(5):510).
7. Lade H, Choudhary SR, Vashishth A. (2014). Endoscopic vs micro $\neg$ scopic myringoplasty: a different perspective. Europ Arch Otorhinolaryngology Jul;271(7):1897-1902).

8. El-Guindy A (1993) Endoscopic transcanal myringoplasty. J Laryngol Otol 106:493-495.

9. Raj A, Meher R (2001) Endoscopic transcanal myringoplasty - a study. Indian J Otolaryngol head neck surg 53(1):47-49.

10. Patel J, Aiyer RG, Gajjar Y, Gupta R, Raval J, Suthar PP (2015) Endoscopic tympanoplasty vs microscopic tympanoplasty in tubotympanic CSOM: a comparative study of 44 cases. Int J Res Med Sci 3(8):1953-1957.

11. Lakpathi G, Reddy LS (2016) Comparative study of endoscope assisted myringoplasty and microscopic myringoplasty. Indian J Otolaryngol Head Neck Surg 68:185.

12. Ghaffar S, Ikram M, Zia S, Raza A(2006) Incorporating the endoscope into middle ear surgery. Ear Nose Throat J.;85(9):593-596.

13. Jyothil A, Shrikrishna B, Kulkarni1 N, Kumar A (2017). Endoscopic Myringoplasty Versus Microscopic Myringoplasty in Tubotympanic CSOM: A Comparative Study of 120 Cases. Indian J Otolaryngol Head Neck Surg (2017) 69(3):357-362.

14. Küçükkavruk L, Temügan E, Kaya M, Unsal S, Gumus N, Yüksel M, Gündüz M (2016). Retrospective air bone gap evaluation of patients with tympanic membrane central perforation. Clin Invest Med 2016; 39 (6): S39-S42. 